\title{
Separação de Fontes Usando o Filtro de Kalman Estendido
}

\author{
Ricardo Suyama, Murilo Bellezoni Loiola, Romis Ribeiro de Faissol Attux e Cynthia Junqueira
}

\begin{abstract}
Resumo-O objetivo deste trabalho é apresentar uma nova proposta para separação de fontes baseada numa formulação de tal problema como uma tarefa de estimação de estado e no uso do Filtro de Kalman Estendido para resolvê-lo. Um dos pontos fortes da metodologia é a maneira natural com que é possível abordar cenários com 1) ruído aditivo; 2) mais fontes que sensores e 3) fontes gaussianas. A avaliação da nova proposta se deu por meio de simulações, no âmbito das quais foi possível buscar termos de comparação com uma técnica que utiliza informação temporal no processo de separação. Os resultados mostram que a idéia é bastante promissora, embora ainda haja um significativo espaço para melhoria, especialmente no que se refere à estratégia de formulação adotada.
\end{abstract}

Palavras-Chave-Separação de fontes, filtro de Kalman estendido, ICA, estimação de estado.

Abstract-The objective of this work is to present a new proposal for blind source separation founded on a formulation of this problem as a state estimation task and on the use of the Extended Kalman Filter (EKF) to solve it. One of the strong points of the methodology is the natural manner whereby it is possible to deal with scenarios with 1) additive noise; 2) more sources than sensors and 3) gaussian sources. The new proposal was tested with the aid of simulations, and it was always a major concern to compare its performance to that associated with a source separation approach that makes use of temporal information. The results show that the idea is quite promising, although there are many possible improvements, particularly insofar as the formulation strategy is concerned.

Keywords-Source separation, Extended Kalman Filter, ICA, state estimation.

\section{INTRODUÇÃO}

Uma mesma tarefa surge em diversos contextos associados à área de processamento de sinais: a de separar fontes de informação tendo por base apenas versões misturadas das mesmas. Tal objetivo é geralmente atingido com a ajuda da diversidade decorrente do uso de múltiplos sensores e de um certo grau de conhecimento a priori acerca das características das fontes.

Há inúmeros problemas de separação de fontes que podem ser formulados nesse espírito, mas, na maioria dos casos, o escopo de aplicação é delimitado com o auxílio de duas hipóteses simplificadoras:

Ricardo Suyama, Murilo B. Loiola e Cynthia Junqueira, Laboratório de Processamento de Sinais para Comunicações, Departamento de Comunicações, Faculdade de Engenharia Elétrica e de Computação, Universidade Estadual de Campinas, Campinas, Brasil, e-mails: \{rsuyama,mloiola,cynthia\}@decom.fee.unicamp.br. Romis R. F. Attux, Departamento de Engenharia de Computação e Automação Industrial, Faculdade de Engenharia Elétrica e de Computação, Universidade Estadual de Campinas, Campinas, Brasil, e-mail: attux@dca.fee.unicamp.br. Este trabalho foi parcialmente financiado pela FAPESP.
1) As fontes são mutuamente independentes;

2) O sistema misturador é linear e instantâneo.

Baseado nestas duas hipóteses, o emprego do ferramental teórico de análise de componentes independentes (ICA, Independent Component Analysis) [1] ao problema de separação de fontes se desenvolveu rapidamente: com tal "abordagem padrão", o campo de pesquisa como um todo atingiu um notável grau de maturidade [2].

Naturalmente, o amadurecimento de uma linha de investigação não é desprovido de "elementos dialéticos": com o passar do tempo, determinadas limitações do modelo clássico de mistura foram apontadas, e, paralelamente a isso, surgiram novas abordagens. É exatamente nesse espírito que se insere este trabalho, dedicado à exposição de uma nova proposta para lidar com o problema de separação em cenários que transcendem o contexto que usualmente tem lugar em ICA.

A base de nossa proposta é formular o problema de separação de fontes como um problema de estimação de estado. Além da motivação natural de trazer para a esfera da área de processamento de sinais um arcabouço teórico amplo e bem-estabelecido, fomos atraídos pela maneira orgânica com que essa formulação permite que sejam incluídos no modelo de sistema três pontos que não são considerados na ICA clássica:

a) A presença de ruído de medida nos sensores;

b) A existência de menos sensores que fontes (caso subparametrizado);

c) A adoção de um modelo gaussiano para as fontes.

A idéia de reescrever o problema de separação de fontes como um de estimação de estado também é feita em [3]. Diferentemente do que propomos neste artigo, onde definimos as fontes como estados, o trabalho em [3] define como estados do sistema as misturas sem ruído, utilizando um filtro de partículas para estimar os estados a partir de misturas ruidosas.

Uma vez formulado o problema de separação como uma tarefa de estimação de estados, restava fazer uma escolha fundamental: a da metodologia que seria adotada para prover estimativas das variáveis de estado a partir dos valores das observações. Nossa escolha recaiu sobre o Filtro de Kalman Estendido (EKF, Extended Kalman Filter) [4], [5], uma ferramenta adequada ao caráter não-linear que, como veremos, emerge da formulação adotada.

De posse desses elementos, passamos à análise do desempenho da metodologia proposta. Para tal fim, foram considerados diferentes cenários, nos quais tivemos por referência uma técnica de separação capaz de lidar com fontes gaussianas: o SOBI [6]. Essa ferramenta, de inspiração bastante distinta, foi por nós considerada uma opção interessante em termos comparativos, uma vez que não temos conhecimento de trabalhos 
com uma linha exatamente igual à aqui exposta no contexto de separação cega de fontes. Alguns outros métodos de separação de fontes empregando filtros de Kalman e suas variações podem ser vistos em [7]-[9]. Em [7], o problema de separação de sinais de voz dentro de um carro é divido em uma filtragem espacial, realizada por um algoritmo de separação que leva em conta o caráter não-estacionário dos sinais de audio, e uma filtragem temporal realizada por um filtro de Kalman. O método proposto em [8] utiliza um filtro de Kalman estendido para adaptar os pesos de um sistema separador formado por uma rede neural auto-organizável. Já em [9], é desenvolvido um filtro de Kalman para branquear as misturas antes de serem separadas usando análise de componentes principais.

Os resultados obtidos indicam que a metodologia apresentada neste artigo é promissora e, em certos casos, bastante eficiente. Por outro lado, também é patente que ainda há melhorias muito relevantes a serem feitas tanto no que se refere à elaboração das equações dinâmicas quanto no que diz respeito ao emprego da ferramenta de estimação.

O trabalho está estruturado da seguinte forma. Na seção II, apresentamos o problema de separação de fontes. Na seção III, expomos os diversos elementos constituintes de nossa proposta, ao passo que, na seção IV, discutimos brevemente os fundamentos da técnica que nos servirá de base para comparação - o SOBI. A seção V, por sua vez, contém os resultados obtidos e sua análise, enquanto a seção VI é dedicada as nossas conclusões.

\section{O Problema de SeParaçÃo de Fontes}

Sejam os sinais $s_{i}(n), i=1, \ldots, N$, um conjunto de fontes e os sinais $x_{i}(n), i=1, \ldots, M$, um conjunto de misturas das mesmas, de modo que:

$$
x_{i}(n)=f_{i}\left[s_{1}(n), \cdots, s_{N}(n)\right], \quad i=1, \ldots, M
$$

sendo $f[\cdot]$ um mapeamento genérico. $\mathrm{Na}$ formulação mais usual do problema de separação de fontes, são adotadas as seguintes hipóteses:

1) As misturas são instantâneas, ou seja, os mapeamentos $f_{i}[\cdot]$ não têm memória.

2) As fontes são mutuamente independentes.

3) O número de misturas é igual ao número de fontes.

4) As misturas correspondem a combinações lineares das fontes.

De posse dessas hipóteses, chegamos à seguinte equação geral:

$$
\mathbf{x}(n)=\mathbf{A s}(n)
$$

Classicamente, o problema de separação, nesse contexto, é resolvido em duas etapas:

a) Define-se um sistema separador (de caráter "inverso" ao sistema misturador) com relação entrada-saída da forma:

$$
\mathbf{y}(n)=\mathbf{W} \mathbf{x}(n) .
$$

b) É proposto um critério para escolha da matriz $\mathbf{W}$ que tenha um caráter não-supervisionado. Tipicamente, esse critério busca uma expressão matemática da independência entre as fontes.

O objetivo final do processo como um todo é chegar a uma situação tal que o vetor de sinais

$$
\mathbf{y}(n)=\mathbf{W} \mathbf{x}(n)=\mathbf{W A s}(n)
$$

seja igual ao vetor $\mathbf{s}(n)$ a menos de fatores de escala e de uma matriz de permutação [1]. Isso é comumente feito com a ajuda de métodos iterativos como o algoritmo FastICA [2].

O modelo apresentado abrange diversos cenários de valor prático; no entanto, há muitos aspectos sistêmicos de grande relevância que não são por ele contemplados. Para os fins do presente artigo, destacamos três:

- A existência de ruído aditivo de medida sobreposto às misturas captadas [10].

- A possível existência de mais fontes que sensores. Nesse caso, a matriz de mistura deixa de ser quadrada, e, de modo geral, deixa de ser possível pensar em soluções lineares que conduzam a um cenário de inversão perfeita.

- A possibilidade de que as fontes tenham um pronunciado caráter gaussiano. Com isso, passa a estar em xeque um dos pilares da ICA: o uso (implícito ou explícito) de estatísticas de ordem superior (EOS) [11] para nortear a escolha da matriz de separação.

Frisamos que a busca por métodos que sejam eficientes nos contextos apresentados anteriormente constitui parte importante do conjunto de "temas de vanguarda" na área de separação de fontes.

Como buscaremos mostrar na próxima seção, uma formulação do problema de separação em termos de espaço de estados permite que essas extensões sejam incorporadas ao modelo de sistema de uma maneira bastante natural, o que, por si só, já seria motivação mais que suficiente para uma investigação desse tipo. No entanto, animou-nos também a possibilidade de contribuir para que o arcabouço conceitual de estimação de estado seja aproximado ainda mais dos problemas de tratamento da informação.

\section{A FormulaçÃo Proposta}

Passemos à análise da metodologia proposta. Nosso ponto de partida, conforme indicado na seção anterior, é um modelo linear e instantâneo com as seguintes características: a) existe ruído aditivo de medida; b) é possível que haja mais fontes que sensores e c) as fontes podem ser gaussianas. De forma simplificada, buscaremos uma maneira de representar o problema de extrair os valores das fontes $s_{i}(n), i=1, \ldots, N$ de um vetor $\mathbf{x}(n)$ com $M$ elementos de acordo com a noção de estimar o estado de um sistema dinâmico a partir de determinadas observações do mesmo.

Para que cheguemos a uma formulação desse tipo, é necessário construir dois sistemas de equações: um conjunto de equações de processo, responsável por indicar a dinâmica associada às variáveis de estado, e uma ou mais equações de medida, que refletem, de certa forma, o tipo de acesso de que se dispõe à informação contida no estado. Matematicamente, se considerarmos que $\xi(n)$ é o vetor que contém as variáveis 
de estado do sistema e que $v(n)$ é o vetor que contém as medidas, teríamos algo como:

$$
\begin{gathered}
\xi(n+1)=\mathbf{F}(n, \xi(n))+\mathbf{r}(n), \\
v(n)=\mathbf{C}(n, \xi(n))+\mathbf{w}(n),
\end{gathered}
$$

onde $\mathbf{F}(n, \xi(n))$ é a matriz de transição de estados, $\mathbf{C}(n, \xi(n))$ é a matriz de medida, ambas possivelmente nãolineares, $\mathbf{r}(n)$ e $\mathbf{w}(n)$ são ruídos descorrelacionados, brancos, de média zero, e com matrizes de correlação $\mathbf{Q}_{1}(n)$ e $\mathbf{Q}_{2}(n)$, respectivamente.

Analisando as equações (5) e (6), podemos chegar a algumas conclusões:

- Tendo em vista que nossa idéia é estimar o estado de um sistema, é tentador associar o estado às fontes, e, nesse caso, a equação de estados deve representar, de alguma maneira, a "regra de evolução temporal" dessas fontes.

- A equação de medida, por sua vez, apresenta uma correspondência natural com o processo de mistura.

Dessa forma, passamos a encarar o mecanismo de separação como um processo de estimar o valor das amostras das fontes tendo em mãos o valor observado das misturas. Essa idéia de tratar o problema de separação como um de estimação estatística surge classicamente em outros contextos da literatura [12].

À luz dessas idéias, surge naturalmente a necessidade de responder a duas perguntas: como seria possível modelar a dinâmica das fontes (de modo a gerar as equações de estado)? Além disso, como fazer com que as equações de medida correspondam às equações do sistema misturador se não conhecemos, a priori, os coeficientes da mistura linear?

Para lidar com o primeiro ponto, optamos por adotar, assim como em [3], um modelo autoregressivo (AR) para as fontes. Esse modelo possui a vantagem de possuir uma dinâmica inerente e de ter uma aplicabilidade significativa em processamento de sinais [5]. É importante notar que, se o ruído de excitação do modelo for gaussiano, as fontes também o serão. Pensando na hipótese fundamental de ICA, usamos excitações independentes para cada uma delas. Também usamos modelos AR distintos para fontes distintas.

O segundo ponto talvez tenha sido o maior problema por nós enfrentado: como lidar com o fato de que os coeficientes da mistura são desconhecidos? A solução adotada, que teve por base um procedimento análogo (embora ligado a um contexto distinto) discutido em [13], foi supor que os coeficientes da matriz A também seriam variáveis de estado, embora a dinâmica a eles associada seja diferente da dinâmica das fontes. Essa metodologia tem a vantagem de permitir que fatores estocásticos e a idéia de variância no tempo sejam incorporados à mistura. Por outro lado, caso o modelo seja estático, é possível que cheguemos a uma dinâmica trivial, como veremos a seguir.

O modelo proposto tem, portanto, a seguinte forma:

$$
\begin{gathered}
s_{i}(n+1)=\alpha_{i} s_{i}(n)+r_{i} \\
a_{j i}(n+1)=a_{j i}(n)
\end{gathered}
$$

Nestas equações, $s_{i}(n), i=1, \ldots N$, correspondem as fontes, $\alpha_{i}$ são parâmetros dos modelos autoregressivos, $r_{i}$ é o ruído de excitação de cada fonte e $a_{j i}, j=1, \ldots M$, são os elementos da matriz de mistura A. Note que o modelo, como esperado, envolve ruído de medida e permite, por construção, que o número de misturas seja diferente do número de fontes.

De posse dessas equações, resta cumprir uma missão fundamental: encontrar um método eficiente para que se possa, a partir das amostras observadas, chegar aos valores do estado em cada momento. Neste artigo, o cumprimento dessa missão é confiado a uma ferramenta clássica na área: o filtro de Kalman estendido (EKF) [4], [5].

Resumidamente, podemos entender o filtro de Kalman (KF, Kalman Filter) [5] como sendo uma metodologia recursiva de estimação de estado. Em outras palavras, o KF permite que se estime, de maneira ótima e iterativa, o vetor de estados num certo instante a partir dos valores derivados da equação de medida. Essa estimação se dá com a ajuda do conhecimento das equações de processo e de medida do sistema dinâmico, bem como das matrizes de covariância dos ruídos envolvidos. Um ponto crucial é a restrição de que todas as equações sejam lineares e que os ruídos sejam processos gaussianos.

Tal restrição faz com que não seja possível aplicar diretamente o filtro de Kalman a nosso problema, pois lidamos com uma equação de medida não-linear. Motivados por isso, decidimos adotar o filtro de Kalman estendido, que utiliza a linearização das equações em torno de pontos relevantes para que se possa empregar todo o ferramental de filtragem de Kalman em certos contextos nos quais ele não poderia ser originalmente aplicado. $\mathrm{Na}$ quadro 1, apresentamos um sumário do algoritmo EKF [5].

Nestas equações, $\mathbf{K}(n, n-1)$ é a matriz de correlação do erro de predição de estados, $\hat{\xi}\left(n \mid \Upsilon_{n-1}\right)$ é o vetor contendo os valores preditos dos estados, no instante $n$, obtidos a partir dos sinais observados do instante inicial até o instante $n-1$ e $\mathbf{F}(n+1, n)$ e $\mathbf{C}(n)$ são as matrizes obtidas da linearização, respectivamente, de $\mathbf{F}(n, \xi(n))$ e $\mathbf{C}(n, \xi(n))$.

\section{EstimaÇÃo de Fontes Baseada EM Estatísticas DE SEgunda ORDEM}

Conforme vimos até aqui, a formulação do problema de separação de fontes utilizando-se o filtro de Kalman emprega a informação sobre o modelo autoregressivo das fontes para construir as equações de estado, e, dessa forma, incorpora a informação temporal das fontes no processo de estimação dos sinais.

A idéia de se explorar a informação temporal dos sinais no problema de separação de fontes também pode ser encontrada em outros algoritmos, como o AMUSE (Algorithm for Multiple Unknown Signals Extraction) [14] e o SOBI (SecondOrder Blind Identification) [6]. Diferentemente dos algoritmos de ICA, estes métodos são capazes de recuperar os sinais baseando-se apenas em estatísticas de ordem 2, isto é, na matriz de correlação dos sinais observados, desde que as fontes possuam espectros distintos. Vejamos como isso é possível.

Suponha que as fontes são independentes entre si (ou pelo menos descorrelacionadas), e que $\mathrm{E}\left[s_{i}(k) s_{i}(k-l)\right] \neq$ 
Quadro 1 Filtro de Kalman Estendido (EKF)

Para $n=1,2, \ldots$

$$
\begin{aligned}
& \mathbf{G}(n)=\mathbf{K}(n, n-1) \mathbf{C}^{H}(n)\left[\mathbf{C}(n) \mathbf{K}(n, n-1) \mathbf{C}^{H}(n)+\mathbf{Q}_{2}(n)\right]^{-1} \\
& \alpha(n)=v(n)-\mathbf{C}\left(n, \widehat{\xi}\left(n \mid \Upsilon_{n-1}\right)\right) \\
& \widehat{\xi}\left(n \mid \Upsilon_{n}\right)=\widehat{\xi}\left(n \mid \Upsilon_{n-1}\right)+\mathbf{G}(n) \alpha(n) \\
& \widehat{\xi}\left(n+1 \mid \Upsilon_{n}\right)=\mathbf{F}\left(n, \widehat{\xi}\left(n \mid \Upsilon_{n}\right)\right) \\
& \mathbf{K}(n)=[\mathbf{I}-\mathbf{G}(n) \mathbf{C}(n)] \mathbf{K}(n, n-1) \\
& \mathbf{K}(n+1, n)=\mathbf{F}(n+1, n) \mathbf{K}(n) \mathbf{F}^{H}(n+1, n)+\mathbf{Q}_{1}(n)
\end{aligned}
$$

$\mathrm{E}\left[s_{j}(k) s_{j}(k-l)\right]$ para $i \neq j$ e algum $l \neq 0$, ou seja, exista um atraso $l$ para o qual os espectros das fontes são diferentes entre si. Além disso, considere que as fontes possuem potência unitária, ou seja, a matriz de autocorrelação dos sinais $\mathbf{R}=\mathrm{E}\left[\mathbf{x x}^{\mathrm{T}}\right]=\mathbf{A} \mathbf{R}_{s} \mathbf{A}^{\mathrm{T}}=\mathbf{A} \mathbf{A}^{\mathrm{T}}$.

O primeiro passo para separar os sinais é realizar um pré-processamento para branquear os dados observados, obtendo, para isso, uma matriz $\mathbf{B}$ tal que $\mathrm{E}\left[\mathbf{B} \mathbf{x} \mathbf{x}^{\mathrm{T}} \mathbf{B}^{\mathrm{T}}\right]=\mathbf{I}$. Dessa maneira reduzimos o modelo (2) a uma mistura $\overline{\mathbf{x}}=$ $\mathbf{B A s}=\mathbf{Q s}$, onde $\mathbf{Q}$ é uma matriz ortogonal, e, com isso, a recuperação das fontes é obtida identificando-se esta matriz de mistura ortogonal.

A matriz ortogonal pode ser obtida a partir da análise da autocorrelação dos sinais observados para um atraso $l \neq 0$. Considere a matriz de autocorrelação para um atraso $l$, dada por

$$
\mathbf{R}(l)=\mathrm{E}\left\{\overline{\mathbf{x}}(k) \overline{\mathbf{x}}(k-l)^{\mathrm{T}}\right\}=\mathbf{Q R}_{\mathbf{s}}(l) \mathbf{Q}^{\mathrm{T}},
$$

onde $\mathbf{R}_{s}(l)$ representa a matriz de autocorrelação dos sinais para um atraso $l$. Como, por hipótese, as fontes são independentes, a matriz $\mathbf{R}_{s}$ será diagonal e apresentará valores distintos, uma vez que os espectros das fontes são diferentes entre si. Dessa forma podemos obter a matriz $\mathbf{Q}$ através de uma decomposição em autovalores e autovetores da matriz $\mathbf{R}(l)$ e assim utilizar $\mathbf{Q}$ para estimar os sinais originais [2].

$\mathrm{O}$ procedimento descrito corresponde ao algoritmo AMUSE, que considera apenas uma matriz de correlação dos sinais observados. Uma extensão deste método é o algoritmo SOBI, que realiza a separação dos sinais utilizando não somente uma matriz de correlação, e sim um conjunto de matrizes referentes a diferentes atrasos. Dessa forma a escolha do atraso ideal torna-se menos crítica, e torna o algoritmo mais robusto a erros de estimação das correlações. Uma vez definido o conjunto de atrasos para os quais serão calculadas as matrizes de correlação, realiza-se uma diagonalização conjunta das matrizes [15], buscando minimizar o funcional

$$
J(\mathbf{W})=\sum_{l} \text { off }\left[\mathbf{W R}(l) \mathbf{W}^{\mathrm{T}}\right]
$$

onde off $[\mathbf{M}]=\sum_{i \neq j} \mathbf{M}_{\mathrm{ij}}^{2}$. Nota-se, portanto, que minimizar o funcional (10) corresponde a minimizar os elementos fora da diagonal das matrizes de autocorrelação. Diferentes métodos de otimização podem ser empregados na minimização de (10). No entanto, neste trabalho, seguimos o algoritmo descrito em [15], que utiliza um algoritmo baseado no método de Jacobi para diagonalização de uma única matriz.

\section{SimulaçÕeS}

Nesta seção, apresentamos um estudo comparativo do desempenho do filtro de Kalman estendido e do algoritmo SOBI. Em todos os cenários simulados, consideramos duas fontes gaussianas independentes, modeladas por processos AR. Uma das fontes possuía pólo em 0.9 e a outra, em -0.9. As fontes foram excitadas por ruídos gaussianos de média nula e variância unitária. As matrizes dos sistemas misturadores foram normalizadas de forma que cada linha da matriz de mistura tivesse norma unitária. A potência do ruído aditivo de medida é definida pela relação sinal-ruído (SNR, Signal to Noise Ratio) na entrada do sistema separador. Além disso, os resultados apresentados nesta seção correspondem à média de 100 realizações para cada SNR.

Para avaliar a qualidade das estimativas $\hat{\mathbf{A}}$ dos coeficientes do sistema misturador, foi utilizada a seguinte métrica [2]:

$E_{1}=\sum_{i=1}^{m}\left(\sum_{j=1}^{m} \frac{\left|p_{i j}\right|}{\max _{k}\left|p_{i k}\right|}-1\right)+\sum_{j=1}^{m}\left(\sum_{i=1}^{m} \frac{\left|p_{i j}\right|}{\max _{k}\left|p_{k j}\right|}-1\right)$,

onde $p_{i j}$ corresponde ao elemento $i j$ da matriz de permutação $\mathbf{P}=\mathbf{B A}$, A é a matriz de mistura e $\mathbf{B}$ é a matriz de separação. No caso do filtro de Kalman estendido, a matriz B é dada por $\hat{\mathbf{A}}^{-1}$. Quanto menor a métrica (11), menor o erro de estimação dos coeficientes do sistema misturador.

No primeiro cenário consideramos receptores com 1 e 2 sensores e matriz de mistura (não normalizada) $\mathbf{A}=\left[\begin{array}{cc}1 & 0.2 \\ 0.9 & 0.4\end{array}\right]$. Na Fig. 1 são mostradas 100 amostras de cada uma das fontes bem como das estimativas feitas pelo filtro de Kalman estendido quando utilizados 2 sensores, enquanto que na Fig. 2 são apresentados os resultados da estimação das duas fontes quando se dispõe de apenas 1 sensor. Já as Tabs. I e II mostram os valores dos erros quadráticos médios (EQM) de estimação de cada fonte usando o algoritmo SOBI, o EKF com 2 sensores (EKF2) e o EKF com 1 sensor (EKF1).

Como podemos notar, o desempenho obtido pelo EKF para a fonte 1 em ambos os casos (1 e 2 sensores) é bastante superior ao do SOBI. Já para a fonte 2, o EKF com dois sensores tem um desempenho melhor que o SOBI para SNRs baixas $(0-5 \mathrm{~dB})$. O EKF volta a ter um desempenho equivalente ao do SOBI para uma SNR de $30 \mathrm{~dB}$. Quando se utiliza apenas 

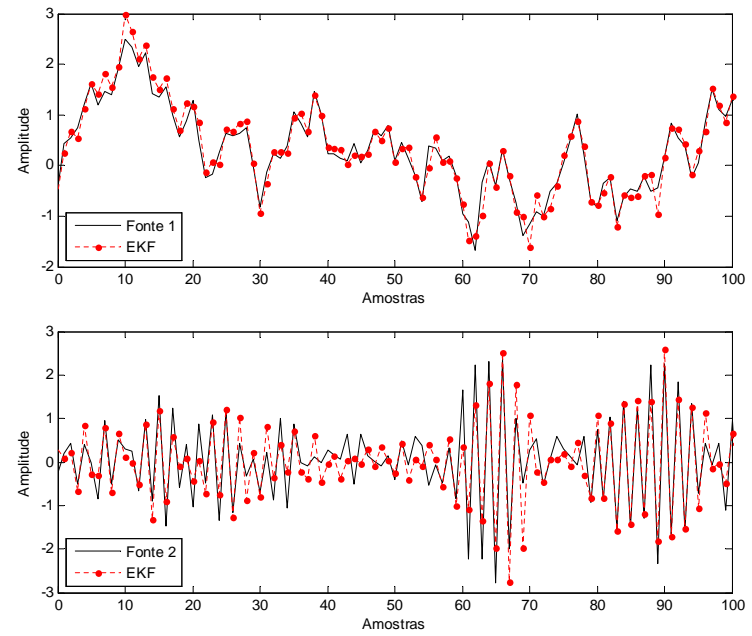

Fig. 1. Desempenho do EKF com 2 sensores para o cenário 1.
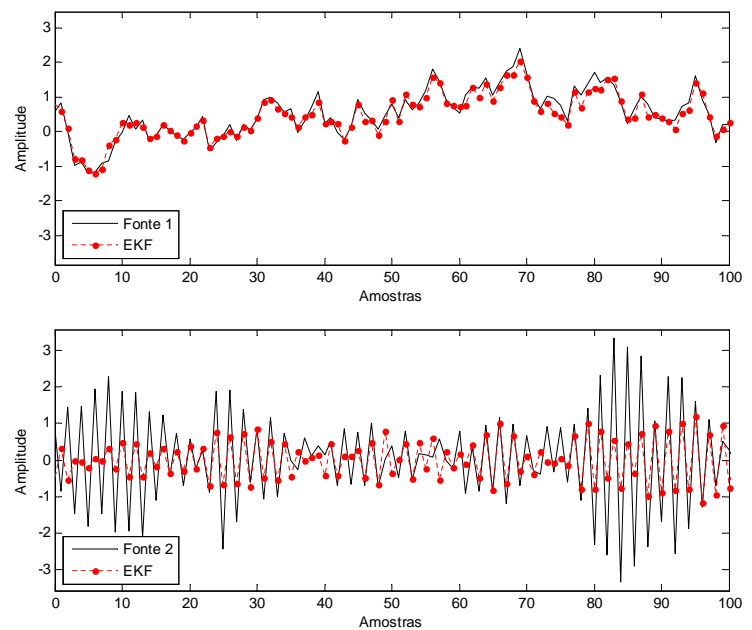

Fig. 2. Desempenho do EKF com 1 sensor para o cenário 1.

TABELA I

ERRO QUADRÁTICO MÉDIO DE ESTIMAÇÃO PARA A FONTE 1 - CENÁRIO 1

\begin{tabular}{|c||c|c|c|c|c|c|c|}
\hline & $0 \mathrm{~dB}$ & $5 \mathrm{~dB}$ & $10 \mathrm{~dB}$ & $15 \mathrm{~dB}$ & $20 \mathrm{~dB}$ & $25 \mathrm{~dB}$ & $30 \mathrm{~dB}$ \\
\hline \hline SOBI & 1,68 & 1,45 & 1,08 & 0,637 & 0,292 & 0,11 & 0,077 \\
\hline EKF2 & 0,24 & 0,12 & 0,06 & 0,035 & 0,019 & 0,01 & 0,004 \\
\hline EKF1 & 0,34 & 0,19 & 0,09 & 0,047 & 0,027 & 0,02 & 0,020 \\
\hline
\end{tabular}

TABELA II

ERRO QUADRÁTICO MÉdIO DE ESTIMAÇÃo PARA A FONTE 2 - CENÁRIO 1

\begin{tabular}{|c||c|c|c|c|c|c|c|}
\hline & $0 \mathrm{~dB}$ & $5 \mathrm{~dB}$ & $10 \mathrm{~dB}$ & $15 \mathrm{~dB}$ & $20 \mathrm{~dB}$ & $25 \mathrm{~dB}$ & $30 \mathrm{~dB}$ \\
\hline \hline SOBI & 1,7 & 0,54 & 0,12 & 0,07 & 0,034 & 0,013 & 0,044 \\
\hline EKF2 & 0,76 & 0,51 & 0,35 & 0,25 & 0,157 & 0,084 & 0,035 \\
\hline EKF1 & 1,26 & 1,01 & 0,92 & 0,83 & 0,813 & 0,724 & 0,667 \\
\hline
\end{tabular}

um sensor (caso subparametrizado), o EKF apresenta um bom desempenho no que se refere à estimação da primeira fonte. A qualidade da estimação da segunda fonte, por sua vez, é significativamente inferior, embora se possa perceber que o sinal obtido acompanha o caráter geral da sequiência desejada. Essa degradação não chega a ser surpreendente, uma vez que há menos sensores que fontes.

A Tab. III mostra os valores da métrica (11) para o SOBI e para o EKF com 2 sensores. É possível observar que os valores calculados para o EKF são menores que aqueles obtidos para o SOBI, indicando que o EKF foi capaz de produzir estimativas mais precisas dos coeficientes da matriz de mistura.

TABELA III

VALOR DA MÉTRICA (11) PARA O CENÁRIO 1

\begin{tabular}{|c||c|c|c|c|c|c|c|}
\hline & $0 \mathrm{~dB}$ & $5 \mathrm{~dB}$ & $10 \mathrm{~dB}$ & $15 \mathrm{~dB}$ & $20 \mathrm{~dB}$ & $25 \mathrm{~dB}$ & $30 \mathrm{~dB}$ \\
\hline \hline SOBI & 1,04 & 1,24 & 0,799 & 0,45 & 0,196 & 0,070 & 0,023 \\
\hline EKF & 0,13 & 0,07 & 0,036 & 0,02 & 0,016 & 0,014 & 0,009 \\
\hline
\end{tabular}

No segundo cenário de simulação consideramos um receptor com 2 sensores e matriz de mistura (não normalizada) $\mathbf{A}=\left[\begin{array}{cc}1 & 0.6 \\ 0.8 & 0.5\end{array}\right]$. A Fig. 3 apresenta 100 amostras de cada uma das fontes bem como as estimativas produzidas pelo filtro de Kalman estendido. As Tabs. IV e V apresentam os valores dos erros quadráticos médios de estimação das duas fontes para o SOBI e para o EKF.
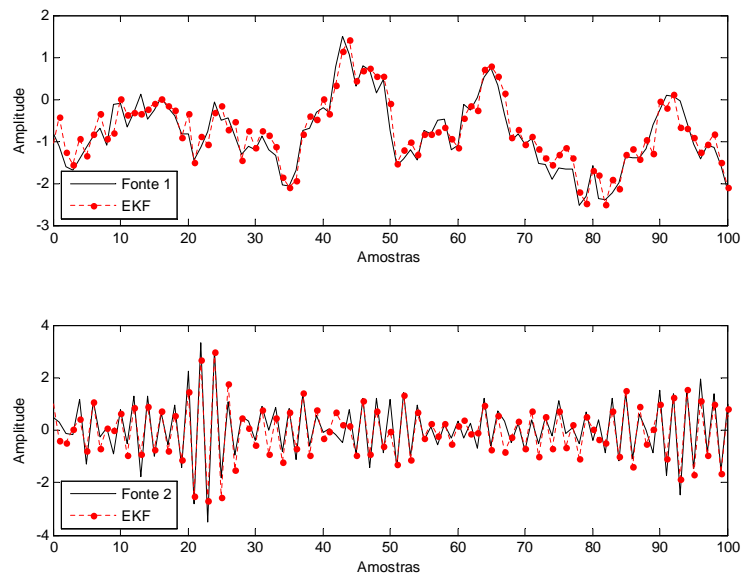

Fig. 3. Desempenho do EKF com 2 sensores para o cenário 2.

TABELA IV

ERRO QUADRÁTICO MÉdIO DE ESTIMAÇÃo PARA A FONTE 1 - CENÁRIO 2

\begin{tabular}{|c||c|c|c|c|c|c|c|}
\hline & $0 \mathrm{~dB}$ & $5 \mathrm{~dB}$ & $10 \mathrm{~dB}$ & $15 \mathrm{~dB}$ & $20 \mathrm{~dB}$ & $25 \mathrm{~dB}$ & $30 \mathrm{~dB}$ \\
\hline \hline SOBI & 1,98 & 1,95 & 1,91 & 1,86 & 1,722 & 1,512 & 1,123 \\
\hline EKF & 0,45 & 0,23 & 0,09 & 0,07 & 0,064 & 0,062 & 0,059 \\
\hline
\end{tabular}

A partir destas tabelas e da Fig. 3 é possível observar que o EKF tem um desempenho melhor que o do SOBI nas estimativas das duas fontes. Aparentemente, para a matriz de mistura usada neste cenário, o algoritmo SOBI não consegue calcular boas estimativas das fontes, independentemente da SNR considerada. O EKF, por sua vez, apresenta valores de 
TABELA V

ERRO QUADRÁTICO MÉdIO DE ESTIMAÇÃO PARA A FONTE 2 - CENÁRIO 2

\begin{tabular}{|c||c|c|c|c|c|c|c|}
\hline & $0 \mathrm{~dB}$ & $5 \mathrm{~dB}$ & $10 \mathrm{~dB}$ & $15 \mathrm{~dB}$ & $20 \mathrm{~dB}$ & $25 \mathrm{~dB}$ & $30 \mathrm{~dB}$ \\
\hline \hline SOBI & 1,14 & 1,04 & 0,98 & 0,97 & 0,976 & 1,004 & 1,156 \\
\hline EKF & 0,62 & 0,31 & 0,22 & 0,18 & 0,173 & 0,165 & 0,161 \\
\hline
\end{tabular}

erro quadrático médio que decrescem bem mais rapidamente com o aumento da SNR.

Por fim, a Tab. VI mostra os valores da métrica (11) para as estimativas dos coeficientes da matriz de mistura calculadas tanto pelo SOBI quanto para o EKF. Aqui também o desempenho do EKF é superior ao do SOBI.

TABELA VI

VALOR DA MÉTRICA (11) PARA O CENÁRIO 2

\begin{tabular}{|c||c|c|c|c|c|c|c|}
\hline & $0 \mathrm{~dB}$ & $5 \mathrm{~dB}$ & $10 \mathrm{~dB}$ & $15 \mathrm{~dB}$ & $20 \mathrm{~dB}$ & $25 \mathrm{~dB}$ & $30 \mathrm{~dB}$ \\
\hline \hline SOBI & 1,00 & 0,98 & 0,97 & 0,971 & 1,045 & 1,191 & 1,531 \\
\hline EKF & 1,32 & 0,93 & 0,50 & 0,269 & 0,127 & 0,085 & 0,047 \\
\hline
\end{tabular}

É importante ressaltar que houve casos em que a metodologia apresentada não teve bom desempenho, especialmente em cenários nos quais os pólos do modelo $\mathrm{AR}$ das fontes tinham o mesmo sinal. Ainda não chegamos a uma explicação plenamente satisfatória para esse fenômeno, mas achamos que o fato de uma configuração de pólos desse tipo levar a sinais com características espectrais semelhantes pode ser indicativo de um elevado grau de complexidade associado à tarefa de separação. Interessantemente, nesses casos, o SOBI pareceu apresentar uma maior robustez. Isso indica, a nosso ver, duas coisas:

a) que ainda é preciso refinar nossa formulação, particularmente no que se refere à dinâmica dos coeficientes da matriz;

b) que pode ser atraente utilizar ferramentas mais poderosas de estimação, como filtros de partículas [4].

\section{CONCLUSÕES}

O principal objetivo deste trabalho foi apresentar uma nova metodologia para resolver o problema de separação de fontes baseada no arcabouço teórico de estimação de estado. A abordagem é construída em duas etapas: primeiramente, fazse necessário obter equações de processo e de medida representativas do problema de separação, para que, em seguida, aplique-se uma ferramenta de estimação apropriada (usamos aqui o Filtro de Kalman Estendido). A metodologia proposta, capaz de abranger de maneira orgânica cenários sistêmicos considerados "difíceis" no âmbito da ICA clássica, foi testada em diferentes ensaios, tendo sempre por pano de fundo uma comparação com um método que explora informação temporal das fontes, o SOBI. Os resultados mostraram que o método é capaz de superar o SOBI em diversos contextos, embora também tenhamos percebido que há configurações sistêmicas nas quais ele não consegue levar a resultados satisfatórios. Nossa apreciação final é que uma abordagem desse tipo é bastante promissora, mas que ainda há espaço para melhorias importantes.

\section{AGRADECIMENTOS}

Os autores gostariam de agradecer à Fundação de Amparo a Pesquisa do Estado de São Paulo - FAPESP - pelo apoio financeiro.

\section{REFERÊNCIAS}

[1] P. Comon, "Independent Component Analysis - a New Concept?" Signal Processing, vol. 36, $\mathrm{n}^{\circ}$. 3, pags. 287-314, 1994.

[2] A. Hyvärinen, J. Karhunen, e E. Oja, Independent Component Analysis. Wiley, 2001.

[3] F. Cong, X. Xu, S. Zhou, S. Du, e X. Shi, "Particle Filtering Based Noisy Blind Source Separation," Electronics Letters, vol. 43, n . 9, 2007.

[4] M. S. Arulampalam, S. Maskell, N. Gordon, e T. Clapp, "A Tutorial on Particle Filters for Online Nonlinear/Non-Gaussian Bayesian Tracking," IEEE Transactions on Signal Processing, vol. 50, $\mathrm{n}^{\circ}$. 2, pags. 174-188, fevereiro 2002.

[5] S. Haykin, Adaptive Filter Theory, $4^{\mathrm{a}}$ ed. Prentice Hall, 2002.

[6] A. Belouchrani, K. Abed-Meraim, J.-F. Cardoso, e E. Moulines, "A Blind Source Separation Technique Using Second-Order Statistics," IEEE Transactions on Signal Processing, vol. 45, $\mathrm{n}^{\circ}$. 2, pags. 434-444, fevereiro 1997.

[7] S. K. Beack, B. Lee, M. Hahn, e S. H. Nam, "Blind Source Separation and Kalman Filter-Based Speech Enhancement in a Car Environment," em Proc. Intelligent Signal Processing and Communication Systems ISPACS04, novembro 2004, pags. 520-523.

[8] S. Todorovic-Zarkula, B. Todorovic, M. Stankovic, e V. Pavlovic, "Extended Kalman Filter in Blind Separation of Nonstationary Signals," em Proc. European Signal Processing Conference - EUSIPCO04, 2004, pags. 1717-1720.

[9] Q. Lv, X.-D. Zhang, e Y. Jia, "Kalman Filtering Algorithm for Blind Source Separation," em Proc. International Conference on Acoustics, Speech, and Signal Processing - ICASSP05, vol. 5, março 2005, pags. 257-260.

[10] A. Cichoki e S. Amari, Adaptive Blind Signal and Image Processing. Wiley, 2002.

[11] C. Nikias e A. Petropulu, Higher-Order Spectra Analysis. Prentice Hall, 1993.

[12] J.-F. Cardoso, "Blind Signal Separation: Statistical Principles," Proceedings of the IEEE, vol. 90, $\mathrm{n}^{\circ} .8$, pags. 2009-2026, 1998.

[13] P. O. Amblard, J. M. Brossier, e E. Moisan, "Phase Tracking: What Do We Gain from Optimality? Particle Filtering vs. Phase-Locked Loops," Signal Processing, vol. 83, $\mathrm{n}^{\circ}$. 1, pags. 151-167, 2003.

[14] L. Tong, R. Liu, e V. Soon, "Indeterminancy and Identifiability of Blind Identification," IEEE Transactions on Circuits and Systems, vol. 38, pags. 499-509, 1991.

[15] J.-F. Cardoso, "Blind Beamforming for non-Gaussian Signals," IEE Proceedings, vol. 140, pags. 362-370, 1993. 